\title{
Multiple solutions and corresponding power output of a nonlinear bistable piezoelectric energy harvester
}

\author{
Arkadiusz Syta ${ }^{1}$, Grzegorz Litak ${ }^{1, a}$, Michael I. Friswell ${ }^{2}$, and Sondipon Adhikari² \\ 1 Faculty of Mechanical Engineering, Lublin University of Technology, Nadbystrzycka 36, 20-618 Lublin, Poland \\ ${ }^{2}$ College of Engineering, Swansea University Bay Campus, Fabian Way, Crymlyn Burrows, Swansea SA1 8EN, UK
}

Received 22 February 2016 / Received in final form 24 February 2016

Published online 13 April 2016

(C) The Author(s) 2016. This article is published with open access at Springerlink.com

\begin{abstract}
We examine multiple responses of a vibrational energy harvester composed of a vertical beam and a tip mass. The beam is excited horizontally by a harmonic inertial force while mechanical vibrational energy is converted to electrical power through a piezoelectric patch. The mechanical resonator can be described by single or double well potentials depending on the gravity force from the tip mass. By changing the tip mass we examine the appearance of various solutions and their basins of attraction. Identification of particular solutions of the energy harvester is important as each solution may provide a different level of power output.
\end{abstract}

\section{Introduction}

The fast development in the miniaturisation of electronics and new trends in monitoring of systems and structures has motivated the development of small scale of small energy harvesting to power these small electronic devices and extend their lifetime and/or remove the need to charge batteries [1]. Ambient vibration energy harvesting is one potential solution. In this field, linear devices are only efficient at resonance, i.e. they are only sensitive to a single frequency are not efficient for variable excitation conditions. On the other hand nonlinear vibration energy harvesters showing broadband frequency transduction are based on nonlinear phenomena. Consequently these material and/or geometrical nonlinearities improve the efficiency of energy harvesting from variable ambient sources via synchronization and nonlinear resonance. However, the nonlinearity also allows the appearance of multiple solutions.

A range of vibration energy harvesting devices have been proposed [2-5]. Recently, in the context of broadband energy harvesting, bifurcations and chaotic vibrations have been studied in several papers. Cao et al. $[6,7]$ studied chaos in the fractionally damped broadband piezoelectric energy generator in a system with additional magnets. Syta et al. [8] analysed the dynamic response of piezoelectric material attached to a bistable laminate plate. In this paper we study the response of a vibrational energy harvester composed of a vertical beam and

\footnotetext{
${ }^{a}$ e-mail: g.litak@pollub.pl
}

a tip mass where the gravitational acceleration introduces a double well potential. This system has shown a number of possible solutions with different power outputs [9]. The appearance of periodic or chaotic solutions, or solutions with single and cross potential well motions, depend on the initial conditions. In this paper we identify these different motions and investigate their dynamics using basins of attraction and Fourier spectrum tools.

\section{The model}

The proposed nonlinear harvester model is a vertical flexible beam of length $L$ with piezoelectric layers and a tip mass, shown schematically in Figure 1. The base is excited in the transverse direction with harmonic base excitation $z(t)=z_{0} \cos \omega t$. The beam carries a concentrated tip mass, $M_{t}$, with moment of inertia, $I_{t}$, at the end of the beam. The horizontal and vertical elastic displacements at the tip mass are $v$ and $u$ respectively, and $s$ represents the distance along the neutral axis of the beam. The model of this system was described and verified experimentally in our previous paper [9].

The beam is assumed to have uniform inertia and stiffness properties, although a non-uniform beam is easily modeled by including the mechanical beam properties in the energy integrals. The beam has cross sectional area $A$, mass density $\rho$, equivalent Young's modulus $E$, and second moment of area $I$.

Assuming a single mode approximation the equation of motion of the beam-mass system is derived in terms of 
Table 1. Parameter values used in the simulation.

\begin{tabular}{lrllrl}
\hline & Beam and tip mass & & Energy harvester & \\
\hline$\rho$ & 7850 & $\mathrm{~kg} / \mathrm{m}^{3}$ & $L_{c}$ & 28 & $\mathrm{~mm}$ \\
$E$ & 210 & $\mathrm{GN} / \mathrm{m}^{2}$ & $b_{c}$ & 14 & $\mathrm{~mm}$ \\
$b$ & 16 & $\mathrm{~mm}$ & $h_{c}$ & 300 & $\mu \mathrm{m}$ \\
$h$ & 0.254 & $\mathrm{~mm}$ & $\gamma_{c}$ & $-4.00 \times 10^{-5}$ & $\mathrm{Nm} / \mathrm{V}$ \\
$L$ & 300 & $\mathrm{~mm}$ & $C_{p}$ & 51.4 & $\mathrm{nF}$ \\
$I_{t} / M_{t}$ & 40.87 & $\mathrm{~mm}^{2}$ & $R_{l}$ & 100 & $\mathrm{k} \Omega$ \\
\hline
\end{tabular}

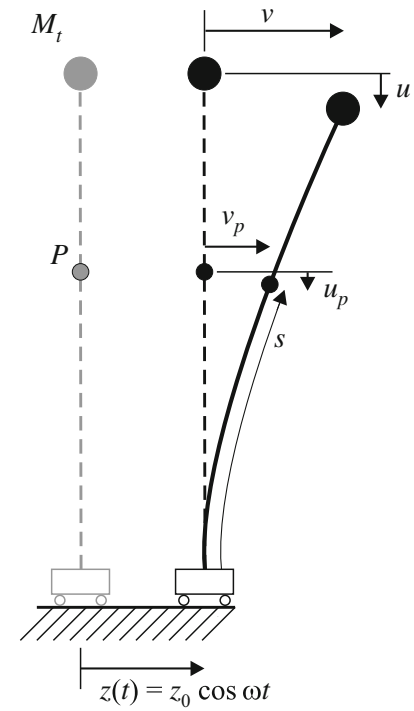

Fig. 1. Schematic representation of the vertical flexible beam with piezoelectric layer and a tip mass. Here, $M_{t}$ denotes the tip mass, $v$ and $u$ are is the horizontal and vertical displacements, $P$ is an arbitrary point on the beam, and $z(t)$ is the harmonic base excitation, while $s$ represents the distance along the neutral axis of the beam.

the displacement of the tip mass using Lagrange's equations [9-11] as:

$$
\begin{aligned}
& {\left[N_{5}^{2} I_{t}+M_{t}+\rho A N_{1}+\left(\rho A N_{3}+M_{t} N_{4}^{2}+N_{5}^{4} I_{t}\right) v^{2}\right] \ddot{v}} \\
& +\left[\rho A N_{3}+M_{t} N_{4}^{2}+N_{5}^{4} I_{t}\right] v \dot{v}^{2}+\left[E I N_{6}-N_{9} \rho A g-N_{4} M_{t} g\right. \\
& \left.+2 E I N_{7} v^{2}\right] v-\Theta_{1} U-\Theta_{2} v^{2} U=-\left[\rho A N_{2}+M_{t}\right] \ddot{z}
\end{aligned}
$$

where $U$ is output voltage. The derivation of this equation and the definitions of the constants are outlined in Appendix. The electrical equation of motion is:

$$
C_{p} \dot{U}+\frac{U}{R_{l}}+\Theta_{1} \dot{v}+\Theta_{2} v^{2} \dot{v}=0
$$

where $C_{p}$ is the capacitance of the piezoelectric patch and $R_{l}$ is the load resistance.

The system is base excited by the harmonic horizontal displacement

$$
z=z_{0} \cos (\omega t)
$$

where $z_{0}$ and $\omega$ are amplitude and frequency of excitation.

The equilibrium positions with no forcing are obtained by setting the velocity and acceleration terms to zero in equation (1) to give

$$
\left[E I N_{6}-N_{9} \rho A g-N_{4} M_{t} g+2 E I N_{7} v^{2}\right] v=0 .
$$

This equation has either one or three solutions, and $v=0$ is always a solution. Since $N_{4}>0$, there are three solutions if

$$
M_{t}>\frac{E I N_{6}-N_{9} \rho A g}{N_{4} g}=M_{t b},
$$

where $M_{t b}$ is the tip mass so that the beam is about to buckle.

We use the same set of system parameters as Friswell et al. [9], as given in Table 1. The post buckled response has two stable equilibrium positions above the critical tip mass, $M_{t b}=10 \mathrm{~g}$.

\section{Numerical solutions}

The energy harvesting system given by equations (1) and (2) is simulated using the Runge-Kutta fourth order method with a fixed time step $\delta t=0.01 \mathrm{~s}$. Figure 2 shows the six different solutions obtained with the system parameters from Table 1 and a tip mass of $M_{t}=10.19 \mathrm{~g}$. Note that our choice of $M_{t}$ implies the beam is buckled with two equilibrium points. The average harvested power, $P$, for a given solution is calculated from the voltage time series, $U_{i}$, by:

$$
P=\frac{1}{N R_{l}} \sum_{i=1}^{N} U_{i}^{2}
$$

where $N$ denotes number of samples.

The different solutions in Figure 2 represent different attractors, obtained from different initial conditions. The excitation amplitude was defined by $z_{0}=16 \mathrm{~mm}$ and the driving frequency was fixed to $0.5 \mathrm{~Hz}$. Attractors (a) and (b) are nonresonant period one single well solutions, and the resulting energy harvested is very low. Attractors (c) and (d) are period three cross well solutions that produce increased harvested power. In contrast solution (e) is chaotic and (f) is a period one resonant solution, both of which give increased power output.

To fully understand the different solutions, Figure 3 shows the corresponding time series, and Figure 4 shows the Fourier spectra, for both displacement and voltage time series. The low energy solutions (a) and (b), and the medium energy solutions (c) and (d), have trajectories that represent the reflections in corresponding equivalent pairs. This is due to the symmetry of the double 

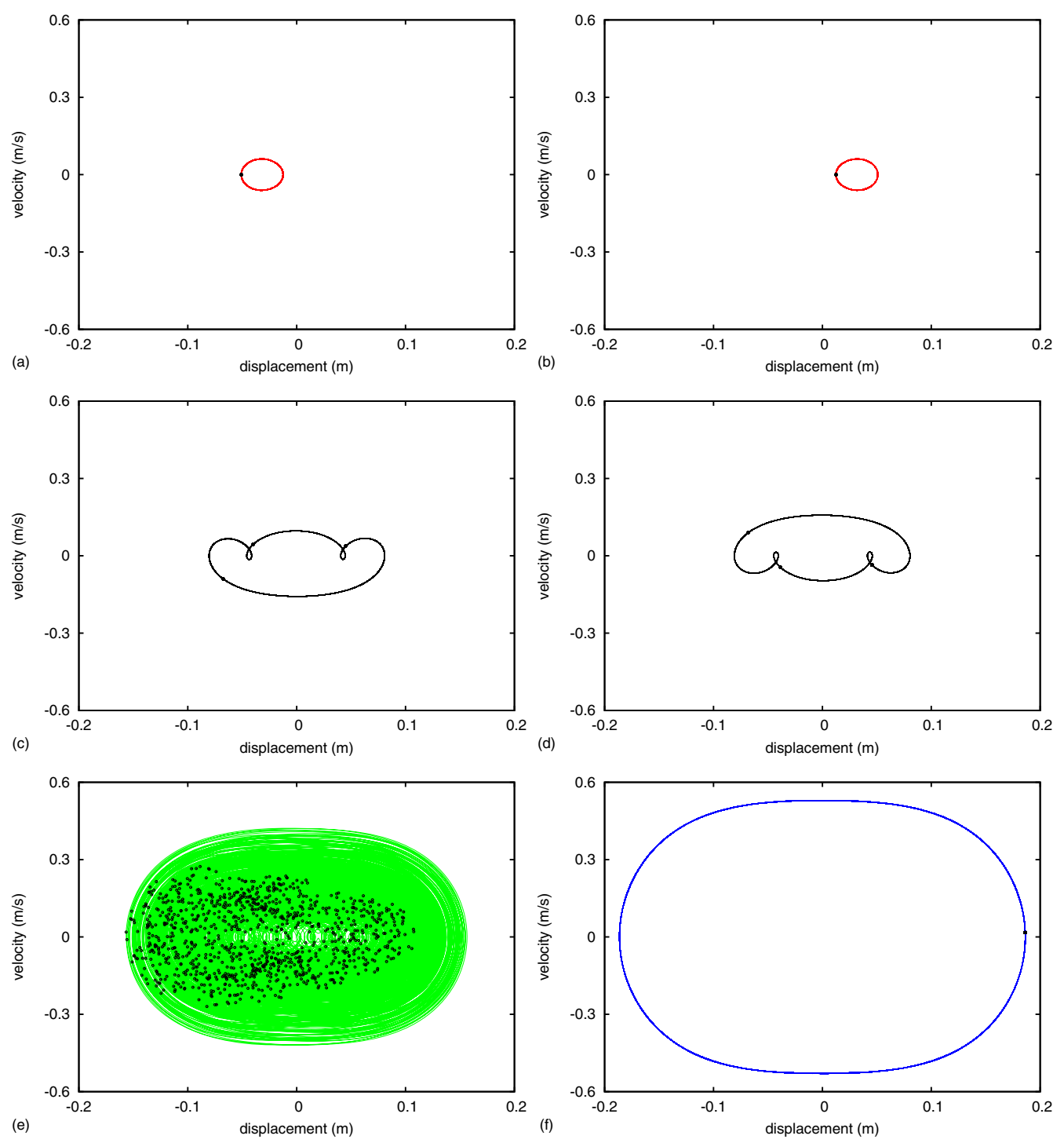

Fig. 2. The response of the system with a tip mass $M_{t}=10.19 \mathrm{~g}$, with kinematic forcing of the fixed amplitude, $z_{0}=16 \mathrm{~mm}$, and frequency, $f=\omega / 2 \pi=0.5 \mathrm{~Hz}$. The lines give the phase portraits, and the points give the corresponding Poincaré sections. The initial conditions and the average harvested power for each case are: (a) $\left(x_{0}, y_{0}\right)=(-0.2990 \mathrm{~m},-0.1805 \mathrm{~m} / \mathrm{s}), P=1.7 \mu \mathrm{W}$, (b) $(-0.2430 \mathrm{~m},-0.2145 \mathrm{~m} / \mathrm{s}), 1.7 \mu \mathrm{W}$, (c) $(-0.2105 \mathrm{~m},-0.2135 \mathrm{~m} / \mathrm{s}), 4.9 \mu \mathrm{W},(\mathrm{d})(-0.2949 \mathrm{~m},-0.1930 \mathrm{~m} / \mathrm{s}), 4.9 \mu \mathrm{W}$, (e) $(-0.2765 \mathrm{~m},-0.1775 \mathrm{~m} / \mathrm{s}), 21 \mu \mathrm{W}$, (f) $(-0.2985 \mathrm{~m}, 0.2399 \mathrm{~m} / \mathrm{s}), 150 \mu \mathrm{W}$.

well potential. More power output can be harvested from the larger trajectories (e) and (f). The Fourier transforms confirm that trajectories (a) to (d) and (f) are regular. The single peaks for solutions (a) and (b) correspond to the excitation frequency. The three discrete peaks for solutions (c) and (d) correspond to the excitation frequency and two subharmonics at $1 / 3$ and $2 / 3$. Interestingly, period three implies chaos [12], and hence it is not surprising that the next solution (e) is chaotic. The non-periodicity is clearly visible in the time series (Fig. 3e) and also in the Fourier transform (Fig. 4e) where discrete peaks change to a continuous spectrum.

A similar mechanical system (without the coupling to the piezoceramic) was investigated by Litak et al. [13]; this system showed Melnikov chaos with Wada patterns of complex mixtures of three basins of attraction. The Wada pattern is a generalization of two mixed areas separated by a fractal border [14]. To investigate this possibility we study the corresponding basins of attraction.

\section{Influence of initial conditions}

To investigate the global dynamical properties of the energy harvester model one can use basins of attraction. In this approach, the stability of particular solutions can be measured by the volume of their basins of attraction, and the borders between them. Consider first the solutions for the harvester with tip mass $M_{t}=10.19 \mathrm{~g}$, 

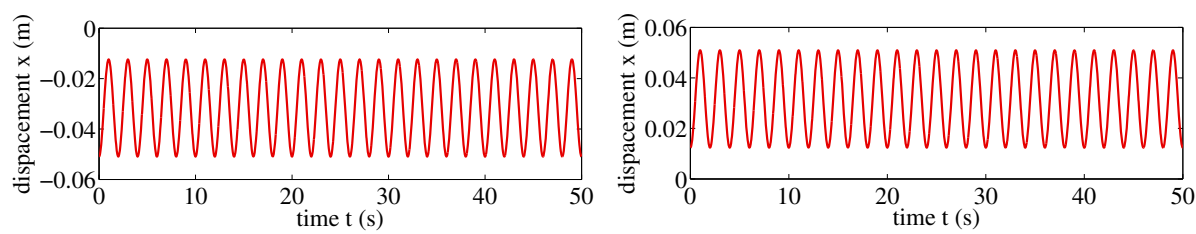

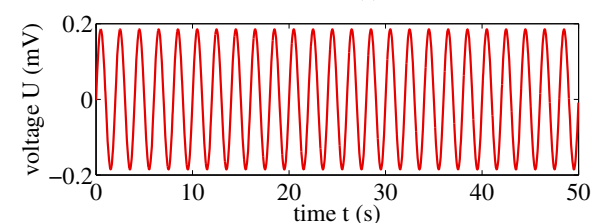

(a)
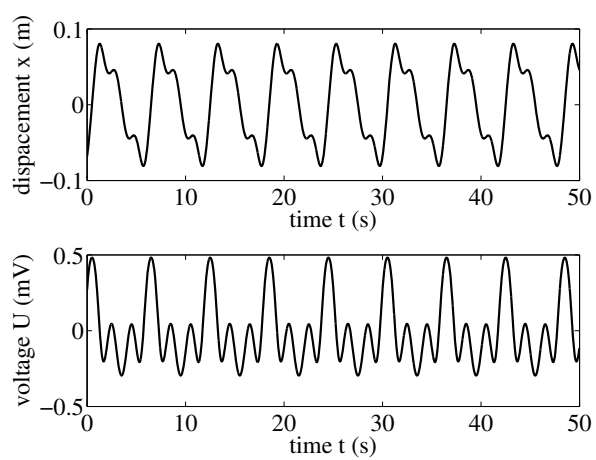

(c)
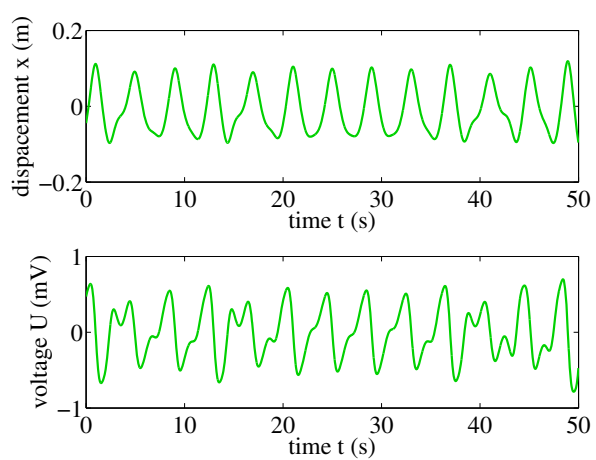

(e)

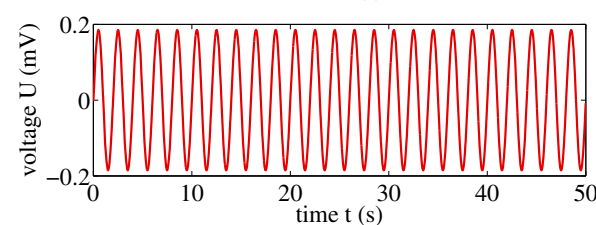

(b)
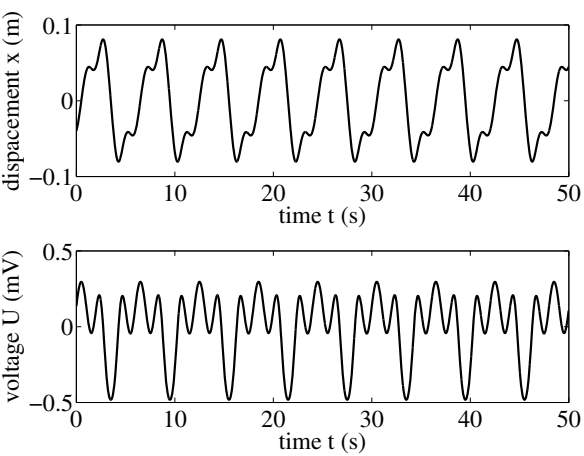

(d)
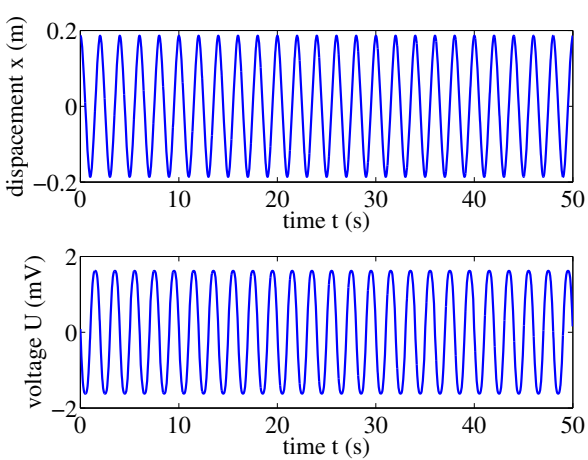

(f)

Fig. 3. Displacement and voltage time series corresponding to the solutions presented in Figure 2.

corresponding to the solutions in Figures 2 to 4. Figure 5 shows the initial conditions that lead to the attractors, which have very different levels of power output. The grid of initial conditions considered in Figure 5 is $\left(x_{0}, y_{0}\right) \in[-0.3,0.3] \mathrm{m} \times[-0.3,0.3] \mathrm{m} / \mathrm{s}$ and $V=0$. The percentage area for the different attractors is as follows: Attractor (f), 61\%; attractor (e), 32\%; attractors (a) and (b), 6.99\%; attractors (c) and (d), 0.01\%. Obviously, the resonant regular attractor (f) is the most common. This attractor has regions of the basin with intensive mixing with other attractors, but also has has a compact region. For small ranges of tip mass displacement and velocities, the single well solutions (a) and (b), are mixed with the regular cross-well solutions (c) and (d), and also with the chaotic cross-well solution (e). Interestingly, large amplitude solutions (f) are also present in these regions. In a larger scale, the compact region of solution (f) has a definite border to the mixed solution regions. Additionally, single well solutions (a) and (b) are most probable in the center of studied area (represented by the two red islands), while (c) and (a) can be classified as rare attractors [15]. Finally, one can distinguish the Wada patterns with the mixture of all attractors (a) to (f) embedded in the compact area of the resonant regular solution (f).

By increasing the tip mass $M_{t}$ to $10.24 \mathrm{~g}$ (Fig. 6) we observe the quantitative change of the basins of attraction plotted in Figure 5 with small changes to the probability of their appearance.

However for $M_{t}=10.39 \mathrm{~g}$ the change is more significant (Fig. 7). The cross well non-resonant solutions (a) 

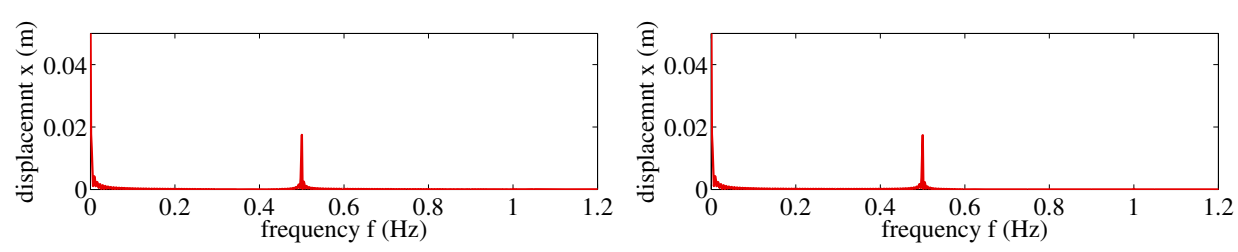

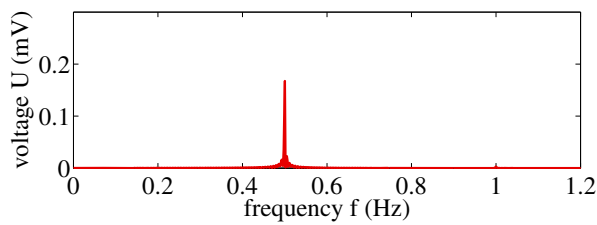

(a)
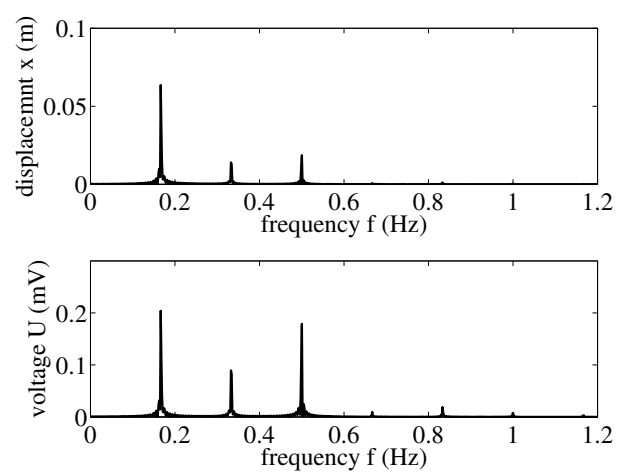

(c)
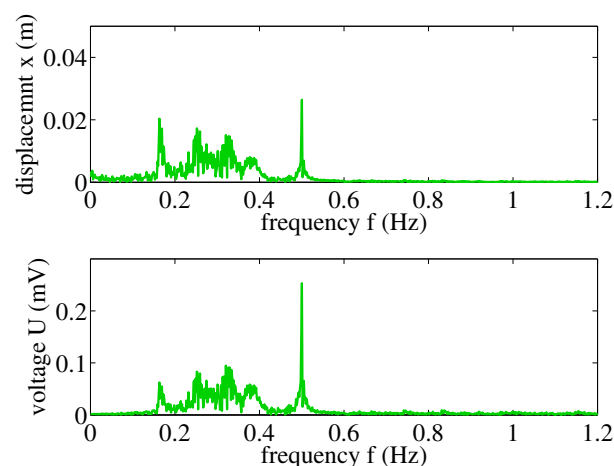

(e)

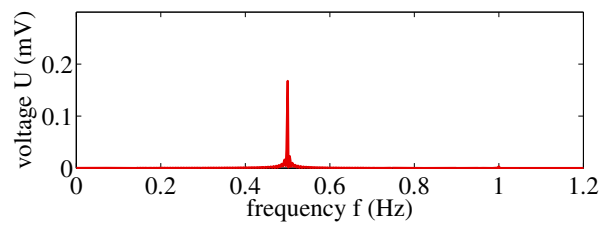

(b)
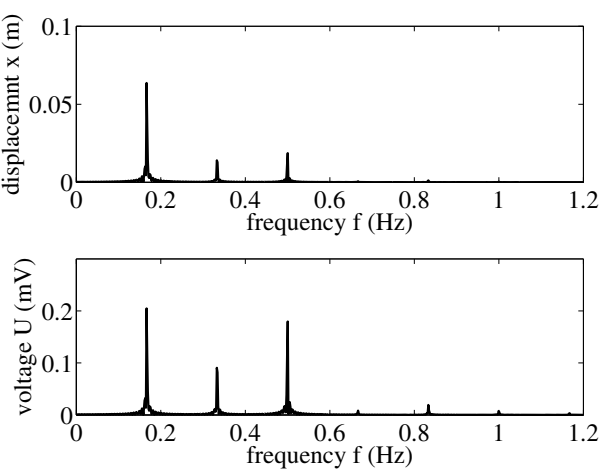

(d)
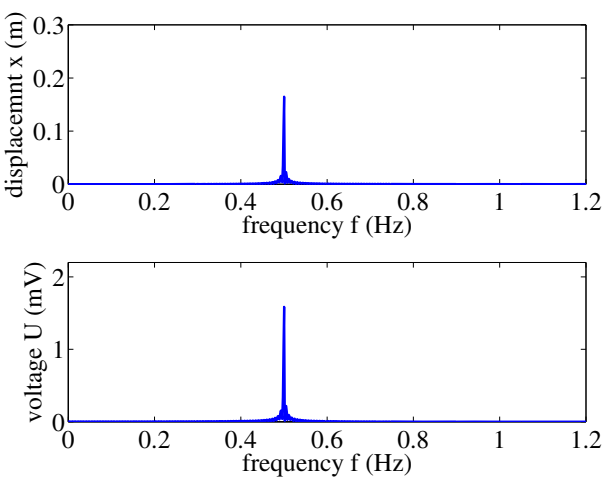

(f)

Fig. 4. Displacement and voltage Fourier spectra corresponding to the solutions presented in Figure 2.

to (d) disappear. Consequently, the percentage appearance of the attractors is now: attractor (f), $57 \%$; and attractor (e), $43 \%$. Note that the Wada pattern is changed to a more simple Melnikov chaos [16] with a fractal border between the two basins of attraction.

\section{Conclusions}

The examined energy harvester exhibited six coexisting vibration responses with regular and chaotic solutions. The power output increased once the system changed from a single well solution to a cross well solution. Further increases in power were related to increases in the size of the trajectory of the tip mass. The corresponding basins of attraction penetrated each other creating Wada patterns with complex mixtures of three or more basins of attraction. We observe these transitions in the basins of attraction by changing the tip mass. The system was excited by an harmonic inertial force at a constant frequency, and the harvester responded at this frequency and also with additional subharmonics dependent on the value of the tip mass.

It is clear that solutions of topologically different attractors would lead to different power outputs in the energy harvesting process. The given basins of attraction enable the stability of the solution to be determined. By combining the distributions of the probability of particular 


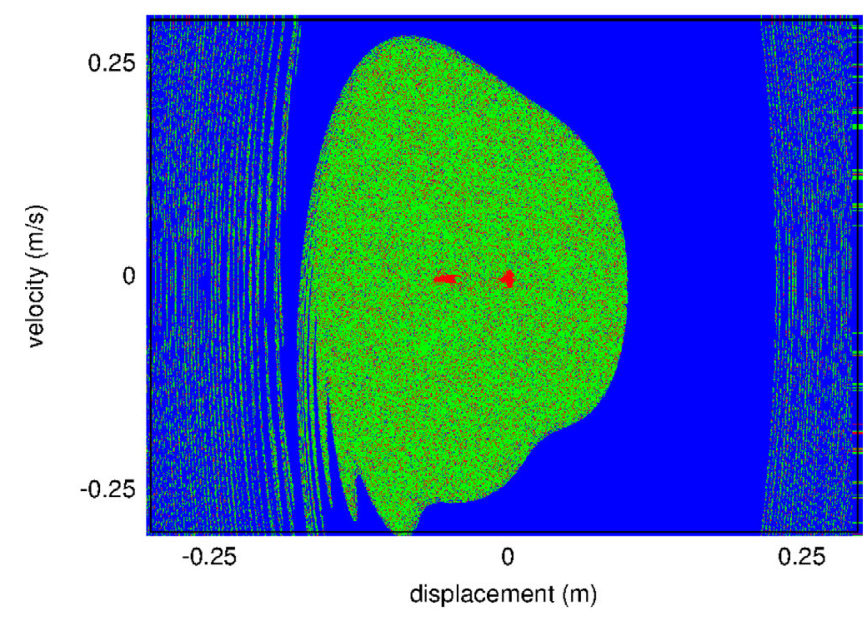

Fig. 5. Basins of attraction calculated for the tip mass $M_{t}=$ $10.19 \mathrm{~g}$ : attractors (a) and (b), red; attractors (c) and (d), black; attractor (e), green; attractor (f), blue.

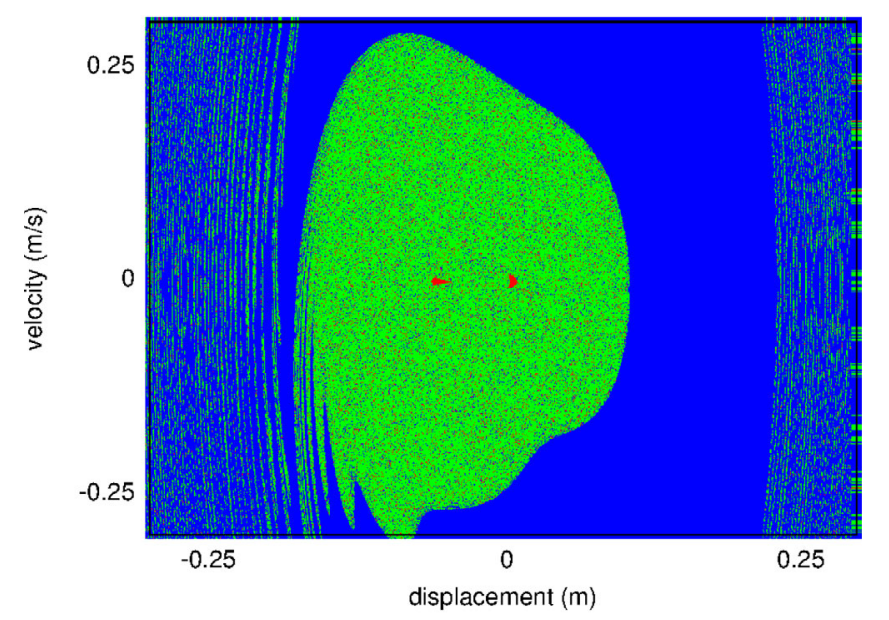

Fig. 6. Basins of attraction calculated for the tip mass $M_{t}=$ 10.24 g: attractors (a) and (b), red; attractors (c) and (d), black; attractor (e), green; attractor (f), blue.

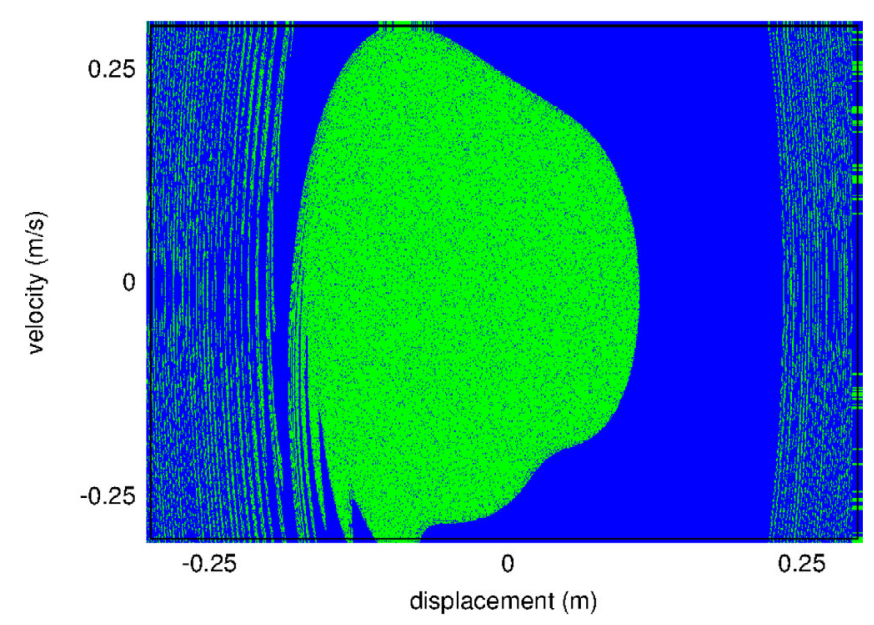

Fig. 7. Basins of attraction calculated for the tip mass $M_{t}=$ 10.39 g: attractor (e), green; attractor (f), blue. solutions occurring, and the associated power output, one can estimate the effective power output of the nonlinear harvester.

GL and AS gratefully acknowledge the support of the Polish National Science Center under grant No. 2012/05/B/ST8/ 00080, and No. DEC-2013/11/D/ST8/ 03308, respectively. The authors acknowledge the support of the Engineering and Physical Sciences Research Council through grant number $\mathrm{EP} / \mathrm{K} 003836$.

\section{Appendix}

We assume the mode shape of the beam (Fig. 1) $\psi(s)$ is

$$
\psi(s)=1-\cos \left(\frac{\pi s}{2 l_{1}}\right) .
$$

With this assumed mode shape, the displacements $v$ and $u$ (Fig. 1) are not independent, and $u$ can be expressed in terms of $v$. Using this single mode approximation [9-11], the kinetic and potential energies of the system in terms of the transverse displacement of the tip mass, $v$, are

$$
\begin{aligned}
T= & \frac{1}{2} \rho A \int_{0}^{L}\left[(\dot{v} \psi(s)+\dot{z})^{2}+\left(v \dot{v} \int_{0}^{s}\left(\psi^{\prime}(\xi)\right)^{2} d \xi\right)^{2}\right] d s \\
& +\frac{1}{2} M_{t}\left[(\dot{v}+\dot{z})^{2}+\left(v \dot{v} \int_{0}^{L_{t}}\left(\psi^{\prime}(s)\right)^{2} d s\right)^{2}\right] \\
& +\frac{1}{2} I_{t}\left[\dot{v} \psi^{\prime}\left(L_{t}\right)+\frac{1}{2} v^{2} \dot{v}\left(\psi^{\prime}\left(L_{t}\right)\right)^{3}\right]^{2} \\
= & \frac{1}{2} \rho A\left[N_{1} \dot{v}^{2}+2 N_{2} \dot{v} \dot{z}+\dot{z}^{2} L+N_{3}(v \dot{v})^{2}\right] \\
& +\frac{1}{2} M_{t}\left[(\dot{v}+\dot{z})^{2}+N_{4}^{2}(v \dot{v})^{2}\right] \\
& +\frac{1}{2} I_{t}\left[N_{5} \dot{v}+\frac{1}{2} N_{5}^{3} v^{2} \dot{v}\right]^{2}
\end{aligned}
$$

and

$$
\begin{aligned}
\Pi= & \frac{1}{2} E I \int_{0}^{L}\left[v \psi(s)^{\prime \prime}+\frac{1}{2} v^{3}\left(\psi^{\prime}(s)\right)^{2} \psi^{\prime \prime}(s)\right]^{2} d s \\
& -\frac{1}{2} \rho A g v^{2} \int_{0}^{L}\left[\int_{0}^{s}\left(\psi^{\prime}(\xi)\right)^{2} d \xi\right] d s \\
& -\frac{1}{2} M_{t} g v^{2} \int_{0}^{L_{t}}\left(\psi^{\prime}(s)\right)^{2} d s \\
= & \frac{1}{2} E I\left[N_{6} v^{2}+N_{7} v^{4}+\frac{1}{4} N_{8} v^{6}\right]-\frac{1}{2} N_{9} \rho A g v^{2} \\
& -\frac{1}{2} N_{4} M_{t} g v^{2}
\end{aligned}
$$


where the beam is assumed to have uniform inertia and stiffness properties. A non-uniform beam is easily modeled by including the mechanical beam properties in the energy integrals. The constants $N_{1}$ to $N_{9}$, are based on the particular integrals of the assumed mode shape $\psi(s)$, and are given by:

$$
\begin{aligned}
& N_{1}=\int_{0}^{L}(\psi(s))^{2} d s=\left(\frac{3 \pi-8}{2 \pi}\right) L \\
& N_{2}=\int_{0}^{L} \psi(s) d s=\left(\frac{\pi-2}{\pi}\right) L \\
& N_{3}=\int_{0}^{L}\left(\int_{0}^{s}\left(\psi^{\prime}(\xi)\right)^{2} d \xi\right)^{2} d s=\frac{\pi^{2}\left(2 \pi^{2}-9\right)}{384} \frac{1}{L} \\
& N_{4}=\int_{0}^{L}\left(\psi^{\prime}(s)\right)^{2} d s=\frac{\pi^{2}}{8} \frac{1}{L}, \\
& N_{5}=\psi^{\prime}(L)=\frac{\pi}{2} \frac{1}{L}, \\
& N_{6}=\int_{0}^{L}\left(\psi^{\prime \prime}(s)\right)^{2} d s=\frac{\pi^{4}}{32} \frac{1}{L^{3}} \\
& N_{7}=\int_{0}^{L}\left(\psi^{\prime}(s) \psi^{\prime \prime}(s)\right)^{2} d s=\frac{\pi^{6}}{2^{9}} \frac{1}{L^{5}} \\
& N_{8}=\int_{0}^{L}\left(\psi^{\prime}(s)\right)^{4}\left(\psi^{\prime \prime}(s)\right)^{2} d s=\frac{\pi^{8}}{4096} \frac{1}{L^{7}} \\
& N_{9}=\int_{0}^{L}\left[\int_{0}^{s}\left(\psi^{\prime}(\xi)\right)^{2} d \xi\right] d s=-\frac{1}{4}+\frac{1}{16} \pi^{2}
\end{aligned}
$$

where $s$ is a variable measured along the beam (Fig. 1).

Similarly, the electromechanical coupling constants (in Eq. (1)) are

$$
\Theta_{1}=\gamma_{c} \psi^{\prime}\left(L_{c}\right) \quad \text { and } \quad \Theta_{2}=\frac{1}{2} \gamma_{c}\left(\psi^{\prime}\left(L_{c}\right)\right)^{3}
$$

where $L_{c}$ is the active length of the piezoelectric material, and $\gamma_{c}$ depends on the properties and arrangement of the piezoelectric material [9].

The Lagrange equations of the second type lead to equations (1) and (2).

\section{References}

1. P.D. Mitcheson, E.M. Yeatman, G.K. Rao, A.S. Holmes, T.C. Green, Proc. IEEE 96, 1457 (2008)

2. M.F. Daqaq, R. Masana, A. Erturk, D.D. Quinn, Appl. Mech. Rev. 66, 040801 (2014)

3. R.L. Harne, K.W. Wang, Smart Mat. Struct. 22, 023001 (2013)

4. S.P. Pellegrini, N. Tolou, M. Schenk, J.L. Herder, J. Intell. Mater. Syst. Struct. 24, 1303 (2013)

5. J. Twiefel, H. Westermann, J. Intell. Mater. Syst. Struct. 24, 1291 (2013)

6. J. Cao, S. Zhou, D.J. Inman, Y. Chen, Nonlinear Dyn. 80, 1705 (2014)

7. J. Cao, A. Syta, G. Litak, S. Zhou, D.J. Inman, Y. Chen, Eur. Phys. J. Plus 130, 103 (2015)

8. A. Syta, C.R. Bowen, H.A. Kim, A. Rysak, G. Litak, Meccanica 50, 1961 (2015)

9. M.I. Friswell, S.F. Ali, O. Bilgen, S. Adhikari, A.W. Lees, G. Litak, J. Intell. Mater. Syst. Struct. 23, 1505 (2012)

10. L. Zavodney, A. Nayfeh, Int. J. Non-Linear Mechanics 24, 105 (1989)

11. E. Esmailzadeh, G. Nakhaie-Jazar, Int. J. Non-Linear Mechanics 33, 567 (1998)

12. T.Y. Li, J.A. Yorke, Amer. Math. Monthly 82, 985 (1975)

13. G. Litak, M. Coccolo, M.I. Friswell, S.F. Ali, S. Adhikari, A.W. Lees, O. Bilgen, in Proceedings of NSC 2012 - 4th IEEE International Conference on Nonlinear Science and Complexity, August 6-11, 2012, Budapest, Hungary, pp. 113-116.

14. J. Aguirre, J.C. Vallejo, M.A.F. Sanjuan, Phys. Rev. E 64, 066208 (2001)

15. M.V. Zakrzhevsky, in Proceedings of the ASME IDETC/EIE, August 30-September 2, 2009, San Diego, California, USA, DETC2009-8665\%, pp. 1411-1418.

16. J. Guckenheimer, P. Holmes, Nonlinear Oscillations, Dynamical Systems, and Bifurcations of Vector Fields (Springer, 1983)

Open Access This is an open access article distributed under the terms of the Creative Commons Attribution License (http://creativecommons.org/licenses/by/4.0), which permits unrestricted use, distribution, and reproduction in any medium, provided the original work is properly cited. 